\title{
Underestimated Burden: Non-Communicable Diseases in North Korea
}

\author{
Shin $\mathrm{Ha}^{1,2}$ and Yo Han Lee ${ }^{3}$ \\ ${ }^{1}$ The Office of Medical Research and Academic Affairs, Korea University Medical Center, Seoul; \\ ${ }^{2}$ Association of Healthcare for Korean Unification, Seoul; \\ ${ }^{3}$ Department of Preventive Medicine, Konyang University College of Medicine, Daejeon, Korea.
}

\begin{abstract}
There remains a misperception that non-communicable diseases (NCDs) are predominantly an issue for developed countries and are not major public health problems in low-income countries. North Korea is also often considered as a country with a disease structure that is typical of a low-income country. Infectious diseases and nutritional problems, including tuberculosis and pneumonia, are still major sources of medical discussion. The authors challenge this misperception by reviewing empirical data on epidemiologic and demographic transitions of North Korea and show that the current NCD burden is the main public health issue in North Korea. In result, it can be said that epidemiologic transition and demographic transition of North Korea preceded prior to economic hardship. It is necessary for the international community, including South Korea, to advance in a new direction of medical support for North Korea.
\end{abstract}

Key Words: North Korea, non-communicable diseases, epidemiologic transition, demographic transition

The burden of non-communicable diseases (NCDs) is thought to be most significant in the populations of wealthy countries; however, evidence suggests otherwise. NCDs are the greatest causes of death in most low and middle-income countries (LMICs), and the socioeconomic and health impacts of NCD in these countries are relatively more severe than in developed countries. ${ }^{1}$ However, the burden of NCDs in LMICs has not received adequate attention from their governments and the international community. ${ }^{2}$ One explanation for this lack of recognition is the generally incorrect perception that NCDs are predominantly an issue for developed countries and are not a major public health problem in LMICs. ${ }^{3}$

North Korea is also often considered a country with a dis-

\footnotetext{
Received: September 20, 2018 Revised: January 29, 2019

Accepted: February 22, 2019

Corresponding author: $Y_{0}$ Han Lee, MD, MPH, PhD, Department of Preventive Medicine, Konyang University College of Medicine, 158 Gwanjeodong-ro, Seo-gu, Daejeon 35365, Korea.

Tel: 82-42-600-6405, Fax: 82-42-600-6401, E-mail: vionic@hanmail.net

-The authors have no potential conflicts of interest to disclose.

(c) Copyright: Yonsei University College of Medicine 2019

This is an Open Access article distributed under the terms of the Creative Commons Attribution Non-Commercial License (https://creativecommons.org/licenses/ by-nc/4.0) which permits unrestricted non-commercial use, distribution, and reproduction in any medium, provided the original work is properly cited.
}

ease structure that is typical of an underdeveloped country. Infectious diseases and nutritional problems, including tuberculosis and pneumonia, are still major sources of medical discussion..$^{4-8}$ In addition, due to the serious health problems following the economic crisis in the mid-1990s, which led to increased poverty, increasing infant mortality, and the emergence of infectious diseases, the international community's preconceptions concerning North Korea have been further confirmed and reinforced. Consequently, international medical assistance to North Korea has hardly addressed NCDs, until now., ${ }^{2,9}$ Despite the commonly held views on North Korean health status, the authors would like to inform readers that North Korea should not be viewed as a typical low-income country by review of empirical data on epidemiologic and demographic transition of North Korea.

Eberstadt, a prominent US policy analyst and a North Korean statistics expert, visited North Korea and obtained the "Democratic People's Republic of Korea Health Statistics, 1987," which contained valuable information on mortality rates in North Korea, prior to the economic crisis. ${ }^{10}$ According to this data, infectious diseases accounted for $29.1 \%$ of the total deaths, and cardiovascular diseases and cancer accounted for only $16.1 \%$ and $2.9 \%$, respectively, up until the 1960 s. In the 1970s, cardiovascular disease emerged as the major cause of 
Table 1. Major Causes of Deaths in North Korea: 1960-2017

\begin{tabular}{lrrrrrrrrrrrr}
\hline \multicolumn{1}{c}{ Major causes } & $\mathbf{1 9 6 0}$ & $\mathbf{1 9 6 5}$ & $\mathbf{1 9 7 0}$ & $\mathbf{1 9 7 5}$ & $\mathbf{1 9 8 0}$ & $\mathbf{1 9 8 5}$ & $\mathbf{1 9 9 0}$ & $\mathbf{1 9 9 5}$ & $\mathbf{2 0 0 0}$ & $\mathbf{2 0 0 5}$ & $\mathbf{2 0 1 0}$ & $\mathbf{2 0 1 7}$ \\
\hline Communicable diseases & 28.3 & 29.1 & 10.9 & 7.5 & 5.2 & 4.0 & 7.6 & 4.9 & 5.3 & 6.7 & 5.5 & 4.8 \\
Cancer & 2.6 & 2.9 & 5.7 & 8.5 & 12.0 & 14.1 & 17.9 & 11.7 & 12.1 & 17.5 & 17.7 & 17.7 \\
Cardiovascular diseases & 12.1 & 16.1 & 22.9 & 32.6 & 42.3 & 45.5 & 30.7 & 20.4 & 22.5 & 34.9 & 36.6 & 38.4 \\
Others & 57.0 & 51.9 & 60.5 & 51.4 & 40.5 & 36.4 & 43.8 & 63.1 & 60.1 & 40.9 & 40.2 & 39.1 \\
\hline
\end{tabular}

Data are presented as a percentage.

Modified until 1985 from Eberstadt and Banister. The Population of North Korea; $1992^{10}$ and since then from the GBD Compare data of The Institute for Health Metrics and Evaluation. ${ }^{17}$

death, overtaking infectious diseases. In the 1980s, approximately $60 \%$ of total deaths were due to cancer and cardiovascular disease (Table 1). The North Korean Ministry of Public Health also reported that, in the period between 1960 and 1991, deaths from cerebrovascular disease increased from $4 \%$ to $25 \%$ of total deaths, and deaths from heart disease increased from $7 \%$ to $18 \% .^{11}$

Epidemiologic transitions are always accompanied by demographic transition, which is usually characterized by reduced mortality and increased life span. North Korea has been no exception. According to UN data, North Korea showed a remarkable decline in mortality rates in the 1960s and 1970s, and the rates of crude deaths in the late 1970s and early 1990s were the lowest in the world, at around five per 1000. ${ }^{12}$ In addition, a dramatic decline in infant mortality and fertility rates, commonly observed in Western societies, and a dramatic increase in infant survival rates were reported in 1990. The average lifespan within North Korea, as estimated by the UN and by Eberstadt, increased to almost the same level as that of South Korea, and the life expectancy of North Korea in 1990 was about the same as that of the upper middle-income countries at that time..$^{10,12}$

From the mid-1990s, North Korea's subsequent general deterioration of living standards and medical systems, stemming from both natural disasters and North Korea's economic crisis, has worsened the health of the North Korea population. In particular, poverty-related deaths, increased rates of infectious diseases, and malnutrition in children were highlighted by the international community. These problems were undoubtedly the underlying cause of North Korea's disastrous public health problems and mortality.

Meanwhile, it is estimated that the adult mortality rate has increased by approximately two to four times during this period. ${ }^{13}$ According to the testimony of many North Korean refugee doctors, the incidence and increase in NCDs, such as cerebral hemorrhage and heart disease, have become a more significant factor in causing deaths. Basic drug therapy and treatment have become impossible, and chronic stress and deterioration in lifestyle have triggered these problems. Alongside this evidence, WHO's North Korean office recently reported that $79 \%$ of deaths were caused by NCDs, of which $36 \%$ were due to cardiovascular disease and $17 \%$ due to cancer. ${ }^{14}$ This figure is comparable to that of high-income countries and, while the data are not robust, they do suggest that NCDs are becoming more of a health burden within North Korea.

It would seem that, while deaths due to nutritional problems and infectious diseases following the economic crisis are gradually decreasing in number, NCDs have also become more problematic. ${ }^{15}$ North Korea exhibits an increasingly aging population and, from the most recent available statistics, has the highest proportion of older adult persons in Southeast Asia (8.7\% in 2008, compared to $8 \%$ in China and 5\% in India). North Korea is also categorized as a country with a high rate of smoking, the most important lifestyle risk factor for NCD. ${ }^{16}$ In addition, the continuing collapse of the current medical system in North Korea is expected to increase the burden of NCD healthcare in North Korea.

Therefore, we suggest that North Korea should not be regarded as a country with an infectious disease-centered death structure that is typical of low-income countries. Rather, it should be considered that there is double burden from deaths caused by NCDs and those caused by communicable diseases. It is necessary for the international community, including South Korea, to advance in a new direction of medical support for North Korea. First, medical support in direct relation to the actual burden of disease should be prepared and provided. Second, in recognition that current health disparities between the two Korean countries can be attributed to NCD burden and differing perceptions, a long-term strategy needs to be developed to allow for a unified approach with which to combat NCD for the long-term benefit of all Koreans.

\section{ACKNOWLEDGEMENTS}

This study was funded by the Korea Foundation for International Healthcare, 2014.

\section{AUTHOR CONTRIBUTIONS}

Conceptualization: Shin Ha and Yo Han Lee. Data curation: Shin Ha and Yo Han Lee. Formal analysis: Shin Ha and Yo Han Lee. Funding acquisition: Yo Han Lee. Investigation: Shin Ha and Yo Han Lee. Methodology: Shin Ha and Yo Han Lee. Project administration: Yo Han Lee. Resources: Shin Ha and Yo Han Lee. Software: Shin Ha and Yo Han Lee. Supervision: Shin Ha and Yo Han Lee. Validation: Shin Ha and Yo Han Lee. Visualization: Shin Ha and Yo Han Lee. Writing_original draft: Shin Ha and Yo Han Lee. Writing—review \& editing: Shin Ha and Yo Han Lee. 


\section{ORCID iDs}

Shin Ha https://orcid.org/0000-0002-1454-731X

Yo Han Lee https://orcid.org/0000-0002-9631-9969

\section{REFERENCES}

1. World Health Organization. Global status report on noncommunicable diseases 2010. Geneva: World Health Organization; 2011.

2. Nugent R, Feigl A. Where have all the donors gone? Scarce donor funding for non-communicable diseases. CGD Working Paper 228. Washington, DC: Center for Global Development; 2010.

3. Siegel KR, Feigl AB, Kishore SP, Stuckler D. Misalignment between perceptions and actual global burden of disease: evidence from the US population. Glob Health Action 2011;4:6339.

4. Chelala C. North Korea: where children look like old men. Lancet 1998;351:1261.

5. Lamar J. Mortality in North Korea rises by $40 \%$ in seven years. BMJ 2001;323:1272.

6. Perry S, Linton H, Schoolnik G. Tuberculosis in North Korea. Science 2011;331:263.

7. McCurry J. No end in sight for North Korea's malnutrition crisis. Lancet 2012;379:602.

8. Amnesty International. The crumbling state of health care in North Korea [accessed on 2019 January 28]. Available at: https:// www.amnesty.org/download/Documents/36000/asa240012010 en.pdf.

9. Grundy J, Biggs BA, Hipgrave DB. Public health and international partnerships in the Democratic People's Republic of Korea. PLoS Med 2015;12:e1001929.

10. Eberstadt N, Banister J. The population of North Korea. Berkeley (CA): Institute of East Asian Studies. University of California, Berkeley; 1992.

11. Korea Foundation for International Healthcare. Mid-to-longterm strategy for North Korean non-communicable diseases. Seoul: Korea Foundation for International Healthcare; 2014.

12. United Nations, Department of Economic and Social Affairs, Population Division. World population prospects: the 2012 revision. Highlights and advance tables. Working Paper No. ESA/P/WP.228. New York (NY): United Nations; 2013.

13. Spoorenberg T, Schwekendiek D. Demographic changes in North Korea: 1993-2008. Popul Dev Rev 2012;38:133-58.

14. World Health Organization. Noncommunicable diseases country profiles 2014. Geneva: World Health Organization; 2014.

15. Lee YH, Yoon SJ, Kim YA, Yeom JW, Oh IH. Overview of the burden of diseases in North Korea. J Prev Med Public Health 2013;46:111-7.

16. Ng M, Freeman MK, Fleming TD, Robinson M, Dwyer-Lindgren L, Thomson B, et al. Smoking prevalence and cigarette consumption in 187 countries, 1980-2012. JAMA 2014;311:183-92.

17. Institute for Health Metrics and Evaluation. GBD Compare (19902017) [accessed on 2019 January 25]. Available at: https://vizhub. healthdata.org/gbd-compare/. 